\title{
Optical pH measurement system using a single fluorescent dye for assessing susceptibility to dental caries
}

\author{
Manuja Sharma \\ Jasmine Y. Graham \\ Philip A. Walczak \\ Ryan Nguyen \\ Lauren K. Lee \\ Matthew D. Carson \\ Leonard Y. Nelson \\ Shwetak N. Patel \\ Zheng Xu \\ Eric J. Seibel
}




\title{
Optical pH measurement system using a single fluorescent dye for assessing susceptibility to dental caries
}

\author{
Manuja Sharma, ${ }^{a}$ Jasmine Y. Graham, ${ }^{b}$ Philip A. Walczak, ${ }^{c}$ Ryan Nguyen, ${ }^{d}$ Lauren K. Lee, ${ }^{e}$ \\ Matthew D. Carson, ${ }^{f, g}$ Leonard Y. Nelson, ${ }^{f, g}$ Shwetak N. Patel, ${ }^{a} Z^{2}$ heng Xu, ${ }^{c}$ and Eric J. Seibel ${ }^{a, f, g, *}$ \\ aUniversity of Washington, Electrical and Computer Engineering, Seattle, Washington, United States \\ bUniversity of California, Berkeley - University of California, San Francisco, Department of Bioengineering, Berkeley, California, United States \\ 'University of Washington, School of Dentistry, Seattle, Washington, United States \\ dUniversity of Washington, Department of Microbiology, Seattle, Washington, United States \\ eUniversity of Washington, Department of Biochemistry, Seattle, Washington, United States \\ fUniversity of Washington, Human Photonics Lab, Seattle, Washington, United States \\ gUniversity of Washington, Department of Mechanical Engineering, Seattle, Washington, United States
}

\begin{abstract}
Sugar-rich diets and poor dental hygiene promote the formation of a biofilm (plaque) that strongly adheres to the dental enamel surface and fosters the evolution of aciduric bacteria. The acid contributes to demineralization of the exterior tooth enamel, which accelerates after the $\mathrm{pH}$ drops below a critical value $(\sim 5.5)$ for extended time periods resulting in the need for restorative procedures. Preventative techniques to alert the dentist and caries-susceptible patients regarding vulnerability to dental decay require a clinical measure of plaque activity. Therefore, there is a need to evaluate the acid production capability of plaque deposits in the pits and fissures of occlusal and interproximal regions. A ratiometric fluorescence $\mathrm{pH}$-sensing device has been developed using an FDA-approved dye and LED excitation. Fluorescein spectral profiles were collected using a spectrometer and analyzed with a spectral unmixing algorithm for calibration over the $\mathrm{pH}$ range of 4.5 to 7 . An in vivo pilot study on human subjects was performed using a sucrose rinse to accelerate bacterial metabolism and to measure the time-dependent drop in $\mathrm{pH}$. The optical system is relatively immune to confounding factors such as photobleaching, dye concentration, and variation in excitation intensity associated with earlier dyebased $\mathrm{pH}$ measurement techniques. () The Authors. Published by SPIE under a Creative Commons Attribution 3.0 Unported License. Distribution or reproduction of this work in whole or in part requires full attribution of the original publication, including its DOI. [DOI: 10 .1117/1.JBO.24.1.017001]

Keywords: fluorescein; anion; dianion; fluorescence; unmixing; caries; $\mathrm{pH}$; plaque; Stephan curve.
\end{abstract}

Paper 180575R received Oct. 5, 2018; accepted for publication Dec. 7, 2018; published online Jan. 8, 2019; corrected Dec. 31, 2020.

\section{Introduction}

Dental plaque formation occurs in a systematic manner, with early bacterial colonizers creating a favorable environment for secondary colonizers. ${ }^{1}$ In the transition from early colonizers to secondary colonizers, the dental plaque microbiome shifts to include a complex mixture of acidogenic (acid-producers) and aciduric (acid-tolerant) species. ${ }^{1-3}$ Plaque bacterial metabolism of dietary sugar and carbohydrates ${ }^{4,5}$ produces lactic and other organic acids as by-products. The $\mathrm{pH}$ of dental plaques drop following a sucrose rinse and is restored back to baseline $\mathrm{pH}$ (now known as the Stephan curve), and repeated time spent below critical $\mathrm{pH}(\sim 5.5)^{6}$ leads to demineralization of enamel. ${ }^{7}$ Organic acids produced by these bacteria, specifically lactic and acetic acid, have been shown to cause enamel demineralization. ${ }^{3,4}$ This demineralization may progress to the clinical diagnosis of dental caries or breakdown of the tooth enamel surface. ${ }^{8}$

As the plaque deposits mature and increase in thickness, the proportion of organic acid-producing bacterial species within the biofilm steadily increases. ${ }^{9}$ The acid-producing bacterial colonies tend to be anaerobic and therefore the biofilm layer adjacent to the enamel surface is exposed to the most acidic

*Address all correspondence to Eric J. Seibel, E-mail: eseibel@uw.edu environment. ${ }^{10}$ In addition, the chemical composition of the extracellular polysaccharide matrix surrounding the bacterial colonies was shown to influence the rate of diffusion of hydronium ions through the biofilm, which may render occlusal fissures susceptible to cariogenic attack by increasing the matrix porosity and diffusion of acid into restricted locations. ${ }^{11}$ Below critical $\mathrm{pH}$, the equilibrium ${ }^{12}$ between enamel demineralization and remineralization is acutely disrupted and early stage caries (white spot) lesions begin to form. For a short time, this intact surface layer ${ }^{13}$ hides the deeper (50 to $100 \mu \mathrm{m}$ ) enamel demineralization. Unless the demineralization process is arrested, the outer surface layer degrades, and a visible opening appears in the enamel surface. If this lesion is detected early, interventions such as fluoride varnish application, etc. can prevent further degradation. ${ }^{14}$ Depending on progression of caries through dentin, more advanced lesions may require invasive restorations to repair structural loss.

A study conducted by Stephan ${ }^{7}$ demonstrated the sudden $\mathrm{pH}$ reduction following a glucose rinse and longer-term recovery to near-neutral $\mathrm{pH}$. Stephan found that subjects with a high propensity for caries exhibited the lowest $\mathrm{pH}$ values following the sugar rinse and required much longer recovery times. Subsequent studies with children and adolescents, using the same sugar rinse approach, yielded a peak and valley Stephan-like timedependent $\mathrm{pH}$ behavior. ${ }^{15-17}$ However, the pediatric studies 
differed in their analysis regarding the correlation of caries incidence to the peak and valley $\mathrm{pH}$ level.

Historically, electrical probe devices have been used to measure the acidic content of a plaque biofilm. ${ }^{7,16,18}$ However, the contact $\mathrm{pH}$ sensor may disrupt the plaque and the readings may not be representative of the acidity of the biofilm that is directly attached to the enamel surface. Depending on the plaque age and nutrients available, the biofilm thickness varies over a wide range from $30 \mu \mathrm{m}$ to over $200 \mu \mathrm{m} .{ }^{19,20}$ Furthermore, typical electrical probes are fragile and require frequent calibration. $\mathrm{pH}$ indicator strips, aka Litmus papers, have also been used to gauge plaque acidity in a sugar challenge study. ${ }^{21}$ However, $\mathrm{pH}$ paper cannot be inserted into the restricted pits and fissures of occlusal surfaces, and the tight interproximal areas between teeth are also challenging. The colorimetric readings of the litmus papers may also be subject to misinterpretation and surface absorbed color-change dyes can leach into the saliva and may result in erratic results. Flexible plastic test strips based on poly(ionic) liquids that anchor the color-sensitive dyes have been developed ${ }^{22}$ but any increase in thickness of these $\mathrm{pH}$ strips would severely limit access interproximally and the occlusal pits and fissures would be unreachable. Confocal laser scanning microscopy (CLSM) has been used to measure the $\mathrm{pH}$ depth profiles of dental bacteria biofilms. ${ }^{19,23}$ CLSM investigations provide a valuable insight into the three-dimensional (3-D) structure of plaque-like biofilms, but adapting this technology to the dental clinic is currently not feasible.

This work explores a new technique of measuring biofilm $\mathrm{pH}$ that can be implemented in vivo using properties of an FDAapproved dye, fluorescein (FL). It is an attractive fluorescent dye that offers high quantum efficiency and is often selected for intensity-based $\mathrm{pH}$ sensing. In addition, FL resides predominantly in the extracellular space (exclusion dye) ${ }^{19,24}$ since it does not penetrate the negatively charged bacterial cell wall. An excitation wavelength near the peak absorption band $(\sim 490 \mathrm{~nm})$ is usually employed and the emission intensity near $520 \mathrm{~nm}$ is recorded. FL emission intensity decreases in a nearly linear fashion as the $\mathrm{pH}$ is reduced. Unfortunately, fluorescence intensity depends upon several factors including the stability of the excitation light source(s), light scattering, dye photobleaching, dye quenching, etc. Overlooked in adapting FL fluorescence to $\mathrm{pH}$ sensing is the change in the relative proportion of its dianion and anion $\mathrm{pH}$-sensitive molecular variants. ${ }^{25,26}$ As the acidity level increases the predominant fluorescent species shifts from dianion to anion. These two species have overlapping absorption and emission spectral characteristics. Unmixing ${ }^{27,28}$ of the overlapping spectral emission data using least-square fitting of the endmember dianion and anion fluorescent species is performed to determine the $\mathrm{pH}$. In addition, a judicious choice of excitation wavelength $(420 \mathrm{~nm})$ makes it possible to balance the stronger dianion absorption/emission with the weaker anion absorption/ emission to optimize the performance of the unmixing algorithm. Other unwanted noise contributions, such as background light and autofluorescence (AF) in the range of 450 to $650 \mathrm{~nm}$, are removed before calculating the biofilm $\mathrm{pH}$.

Therefore, using the spectral unmixing algorithm in combination with the FL fluorescent dye, we achieved many of the desirable features of a ratiometric dental $\mathrm{pH}$ sensor such as operation with a single excitation wavelength, noncontact, and immunity from changes in dye concentration, excitation intensity, and photobleaching. A fiber optic probe was developed that can measure acidity levels in difficult to access dental locations such as occlusal pits and fissures. Details of the construction of the fiber optic $\mathrm{pH}$ probe, calibration methods, spectral unmixing algorithm, and first-in-human pilot study are presented.

\section{Methods and Materials}

\subsection{Chemistry}

\subsubsection{Preparation of fluorescein solutions}

1-M stock solution of sodium FL (Sigma Aldrich and ScienceLab) was prepared in deionized water that was diluted to make FL solutions. Buffered FL solutions were used for calibration of the $\mathrm{pH}$ device with a conventional $\mathrm{pH}$ meter (ThermoFisher Scientific). FL was diluted in phosphate citrate buffer (0.2-M dibasic sodium phosphate, 0.1-M citric acid, $\mathrm{pH}$ indicated for each experiment), 0.1-M sodium bicarbonate buffer, or chemically defined medium (CDM) buffer. ${ }^{29}$ FL solutions were stored in test tubes and wrapped in aluminum foil and handled with minimal light exposure. We used different concentrations of the FL solutions ranging from 100 to $600 \mu \mathrm{M}$.

\subsection{Hardware}

An Ocean Optics spectrometer (USB 2000+) equipped with Ocean View software recorded the fluorescence spectral profile. A pulsed 420-nm LED housed with a driver (ThorLabs, M420F1) served as the FL excitation source. The pulsed LED and spectrometer were synchronized using an Arduino board controlled via computer. A bandpass filter (Semrock, FF01-425/26-25) centered at a 425-nm limited the bandwidth of the LED emission and also attenuated the yellow-green LED defect emission centered near $550 \mathrm{~nm} \cdot{ }^{30}$ A $450-\mathrm{nm}$ long pass filter (Semrock, BLP01-458R-25) blocked the strong blue LED emission from entering the spectrometer and distorting the FL fluorescence spectrum. The 1-in. diameter filters were mounted into an in-line holder (Mightex, SPC-FTH-V1). Dual fiber optic cables were used to collect the fluorescence emission as well transmit the LED excitation. The configuration shown in Fig. 1 was used for calibration with FL buffers in a 1-mm glass cuvette (Firefly, GL) at a distance of $3 \mathrm{~mm}$ from the light collection/transmitting fiber optic probe (ThorLabs, BFY200HS02). The custom dental probe was used for in-vitro testing of tooth samples and in vivo plaque $\mathrm{pH}$ measurement. An optical power meter (Newport \#1830-C) was used to measure the LED output from the probe tip. Due to losses in the optical fiber cables and connectors the output power was in the range of 40 to $180 \mu \mathrm{W}$. 500- $\mu \mathrm{m}$-diameter plastic fiber optics (Fiberfin, FF-SK-20) in the dental probe device were mounted inside a 3-D printed, nonfluorescent black plastic (Hatchbox PLA) holder. Based on the shapes of conventional dental tools, the probe incorporated a 90-deg turn at the tip to simulate clinical dental tools and had a form factor of a toothbrush. Special care was taken in selecting the materials for the probe to avoid unwanted AF. A food-grade silicone molded hood covered the probe tip to reduce ambient light interference. The silicone was made completely black by adding iron oxide powder, $0.3-\mu \mathrm{m}$ particle size, in the molding mixture. The hood was replaced in lieu of clinical hygiene requirements along with cleaning the probe with disinfectant wipes (Metrex Cavicide). In addition, a disposable plastic sheath was used to cover the probe with openings for the fiber optics. 


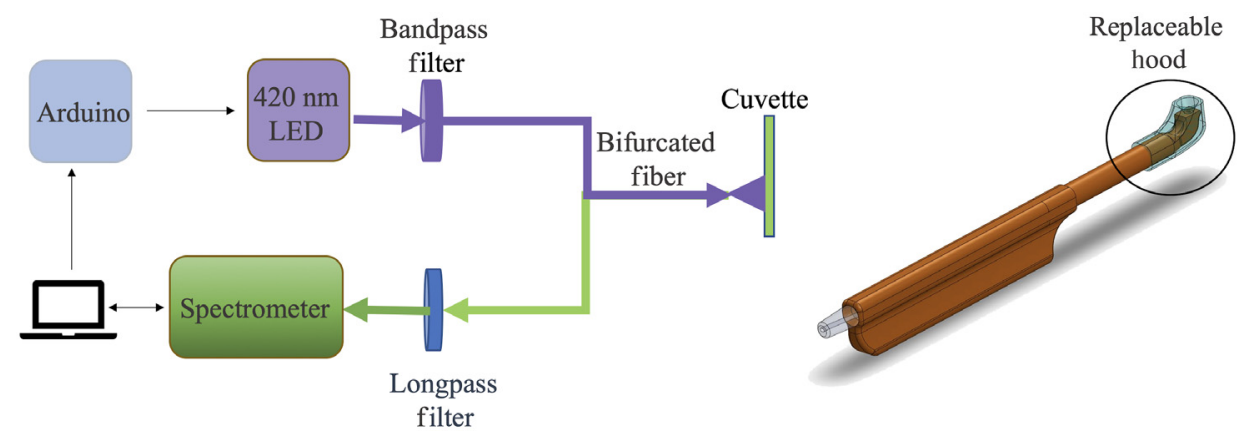

Fig. 1 (a) Diagram of electro-optical device and (b) dental probe.

\subsection{Algorithm}

\subsubsection{Calibration curve: linear unmixing}

In aqueous solution FL exhibits an equilibrium mixture of four different species (cation, neutral, anion, and dianion) that depends upon $\mathrm{pH}$. Only the dianion and anion species are fluorescent. ${ }^{25}$ With increasing $\mathrm{pH}$ of an FL solution, the anion concentration decreases and the dianion concentration increases. For example, at a $\mathrm{pH} 4$, an FL solution will consist of predominantly anions (A) and at a $\mathrm{pH} 9$, the solution will contain mainly dianions (D) resulting in different spectral properties in the 450- to 650-nm range [Fig. 2(a)]. Solutions between $\mathrm{pH} 4.5$ and 7 contain both dianion and anion species resulting in a fluorescent spectral profile that is a mixture of individual emission profiles. Using the spectral properties of endmembers (pH 4 and 9) for pure anion and dianion species, we performed a linear unmixing of the normalized spectral profiles (S) for unknown $\mathrm{pH}$ solutions in the 4.5- to 7-range as

$S_{\mathrm{pH} \_ \text {unknown }}=D * S_{\mathrm{pH} 9}+A * S_{\mathrm{pH} 4}$.

The relative proportion of anions (A) and dianions (D) in the solution was obtained using a least mean square (LMS) regression. Using nine samples between $\mathrm{pH} 4.5$ and 7, we obtained two $\mathrm{pH}$ calibration curves, one for the dianion fraction in the solution and other for the anion fraction as described in Sec. 3. For this analysis, the recorded spectrum was normalized using the peak intensity value in the spectrum range.

\subsubsection{Mitigation of tooth $A F$}

Tooth enamel exhibits a broad fluorescence starting at $450 \mathrm{~nm}$ and extending to $700 \mathrm{~nm}$ with a peak around $500 \mathrm{~nm}^{25}$ A blue 420-nm LED will excite this AF and contribute a noise source to the FL emission profile as shown in Fig. 2(b). To remove this AF interference, we first measured the AF of the tooth before measuring the FL emission. We then subtracted the AF from the total FL spectrum using a curve fitting algorithm and assumed that the AF was the predominant emission feature in the 460- to 480$\mathrm{nm}$ range. Hence, we scaled the entire AF patient tooth profile with the limited spectral signal using the LMS function to obtain the overall AF spectral contribution. The corrected FL emission (Denoised FL) profile was then renormalized as shown in Fig. 2(c).

\subsubsection{Determination of plaque $\mathrm{pH}$}

Plaque $\mathrm{pH}$ was measured using the FL fluorescence methodology for in vivo pilot study. Fluorescence data were collected and processed as described above and summarized in Fig. 3. Ambient light spectrum was collected in addition to AF and FL spectra to perform background light subtraction.

\section{Experiments and Results}

\subsection{Calibration}

Using a 1-mm glass cuvette and the bifurcated fiber optic positioned $3 \mathrm{~mm}$ from the cuvette [Fig. 1(a)], we measured nine different $200-\mu \mathrm{M}$ FL buffers from $\mathrm{pH} 4.5$ to 7 . Each measurement

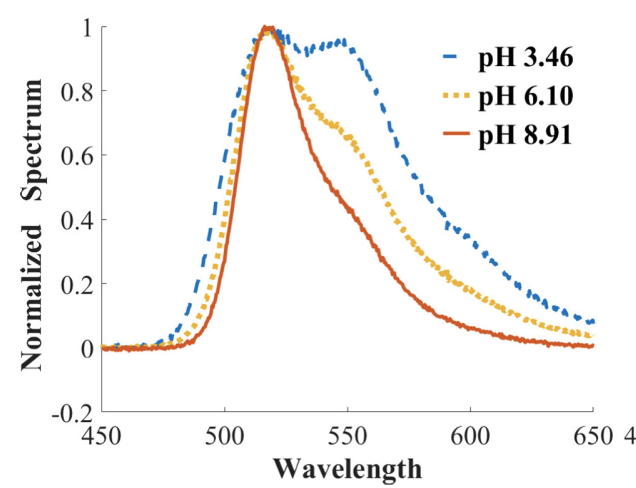

(a)

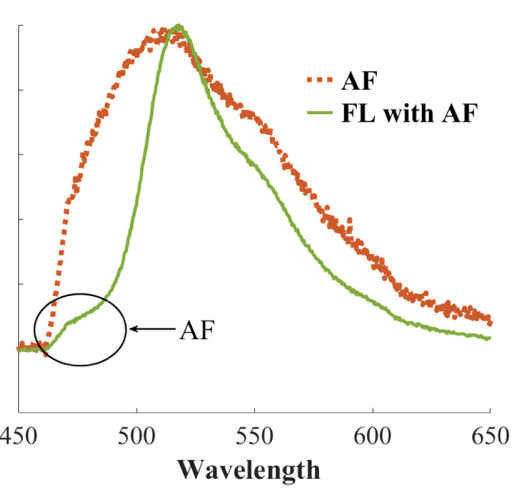

(b)

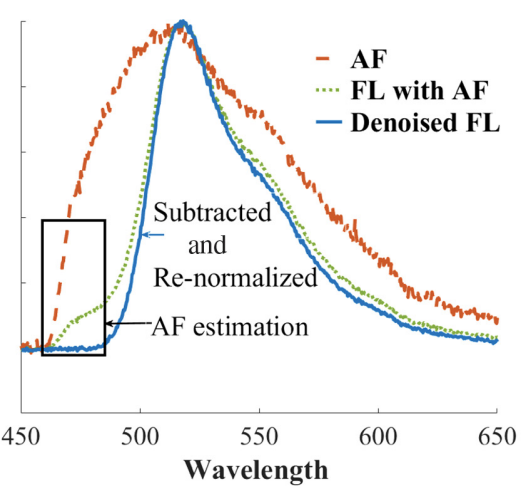

(c)

Fig. 2 (a) FL emission spectrum ( 450 to $650 \mathrm{~nm}$ ) for different pH solutions. (b) Tooth AF using 420-nm LED and FL spectrum with noisy AF (c) AF removal. All signals have been normalized using the peak intensity recorded. 


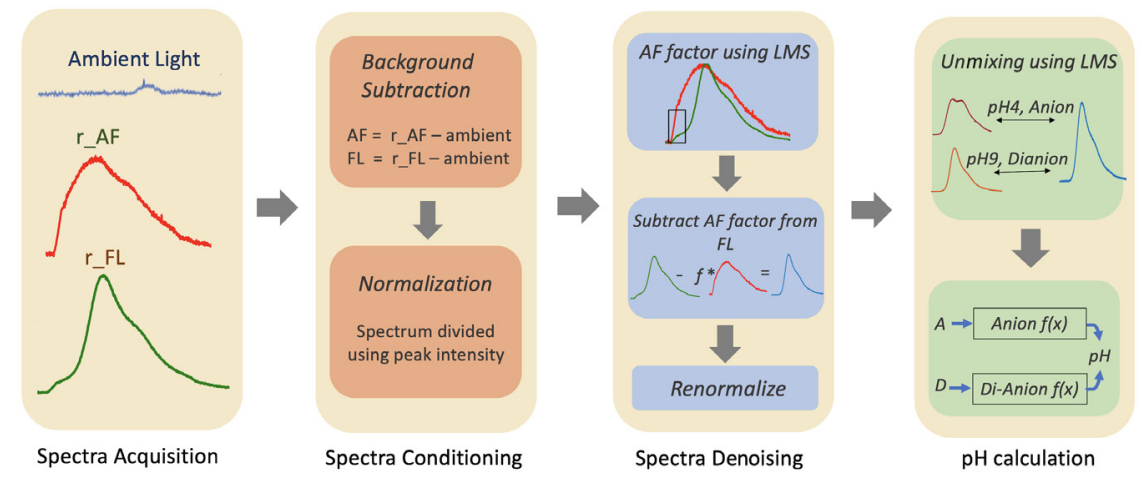

Fig. 3 Procedure for plaque $\mathrm{pH}$ measurement.

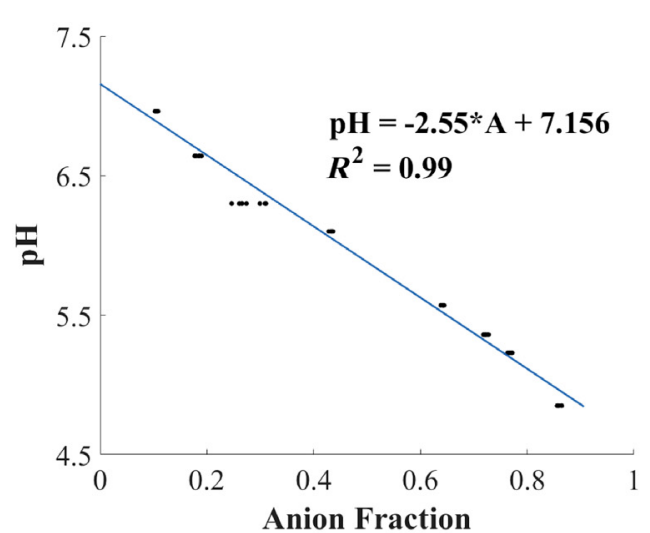

(a)

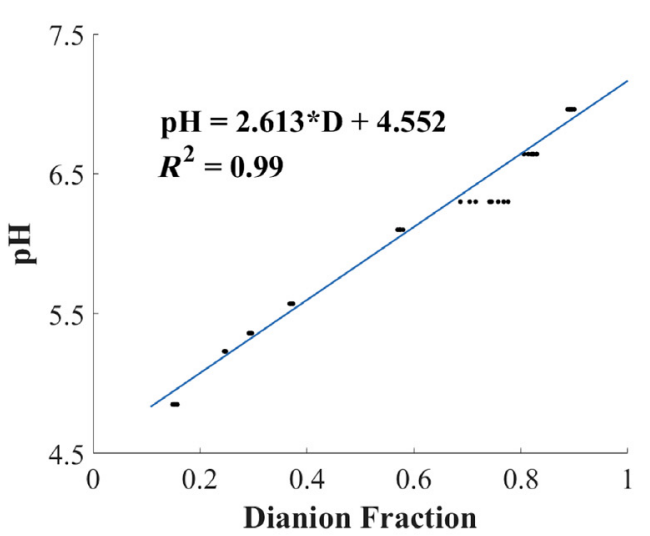

(b)

Fig. 4 Calibration curves obtained using 200- $\mu \mathrm{M}$ buffered FL solution in 1-mm glass cuvette. (a) Anion calibration curve and (b) dianion calibration curve.

was repeated 10 times to obtain the anion and dianion calibration curves using linear unmixing as shown in Fig. 4. Each of the calibration equations were used to predict $\mathrm{pH}$ in the range of 4.5 to 7 .

\subsection{Verification}

\subsubsection{Buffer samples}

We created a test set consisting of different buffer values than those used for calibration in the same $\mathrm{pH}$ range. We also varied the concentration of FL from 100 to $600 \mu \mathrm{M}$ solution and the measurement distance of the cuvette and bifurcated fiber ranging from 1 to $3 \mathrm{~mm}$. Figure 5 shows a Bland Altman plot using 95\% limit of agreement between the value measured with the gold standard $\mathrm{pH}$ meter and derived using our FL method [Eq. (1)]. Using the anion calibration curve, the root mean square error (RMSE) of 100 samples is $0.14 \mathrm{pH}$ and one standard deviation (SD) is $0.14 \mathrm{pH}$. By comparison, using the dianion calibration curve the RMSE is $0.2 \mathrm{pH}$ and SD is $0.15 \mathrm{pH}$.

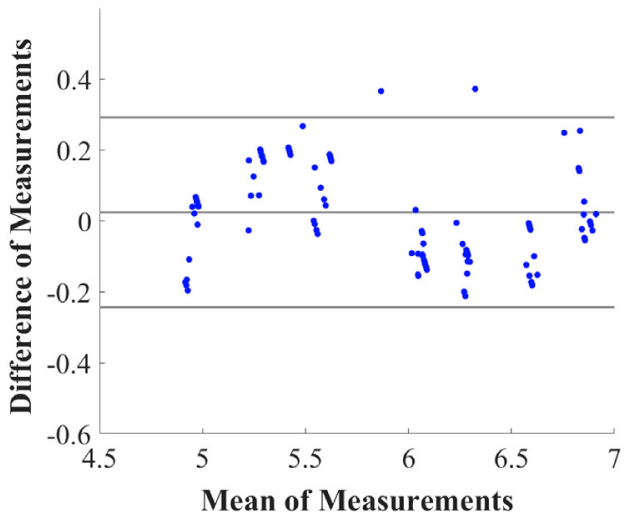

(a)

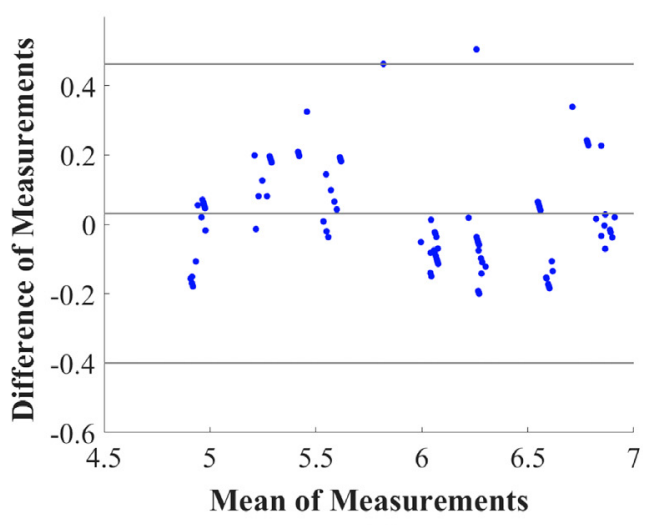

(b)

Fig. 5 Bland-Altman plot (with $95 \%$ agreement) between reference $\mathrm{pH}$ meter and predicted $\mathrm{pH}$ values using FL for (a) anion and (b) dianion. 


\subsubsection{In vitro tooth samples}

To confirm that AF can be removed from the FL spectrum to predict $\mathrm{pH}$ values, we tested buffered FL for different $\mathrm{pH}$ values on two extracted molars. Surgically extracted (January 2018) impacted third molars were obtained for in vitro study. Samples were refrigerated in $1 \%$ Thymol and washed with distilled water before testing. We first recorded the ambient background and AF spectra and then applied a $10-\mu \mathrm{L}$ drop of buffered FL to the tooth surface (occlusal) and recorded the resultant spectrum. All the measurements with the dental probe [Fig. 1(a)] were taken with a separation distance of $2 \mathrm{~mm}$ between the tip of the probe and the FL drop. Teeth were rinsed using water between measurements. The result using the anion calibration curve for two extracted teeth is represented in Fig. 6, we used 200- $\mu$ M FL for testing. Three measurements were recorded for each buffer value. RMSE and one SD for 24 samples were 0.3 and $0.2 \mathrm{pH}$, respectively.

\subsection{Pilot Study: Resting ph and Sucrose Response}

To demonstrate the clinical utility of the FL-based optical $\mathrm{pH}$ system, we measured the resting $\mathrm{pH}$ (without sucrose) and then monitored the $\mathrm{pH}$ response of a $0.3-\mathrm{M}$ sucrose rinse in eight subjects. An equal number of male and female subjects participated in the study with the median age of 23.5 years and mean age of 28 years. Two of the eight subjects voluntarily skipped brushing the day of the study. For testing, a dentist selected one tooth surface in each of the four different quadrants. She used visual inspection to select surfaces with high plaque load while avoiding interproximal spots. For the resting $\mathrm{pH}$, we recorded three spectra: ambient light, AF, and FL applied to the tooth. Each of the readings was repeated twice to measure repeatability. FL $(400 \mu \mathrm{M})$ was applied using an irrigation syringe like the one shown in Fig. 7.

Subjects then rinsed their mouth to remove any FL and then rinsed and retained $10 \mathrm{ml}$ of $0.3-\mathrm{M}$ sucrose solution for $15 \mathrm{~s}^{17}$

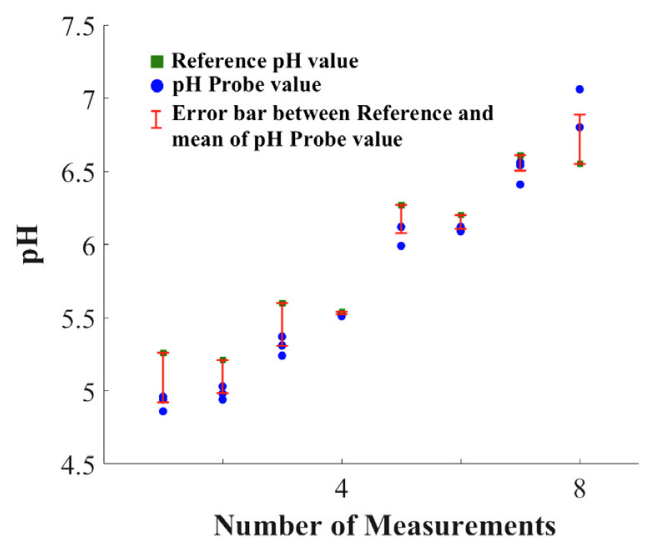

(a)

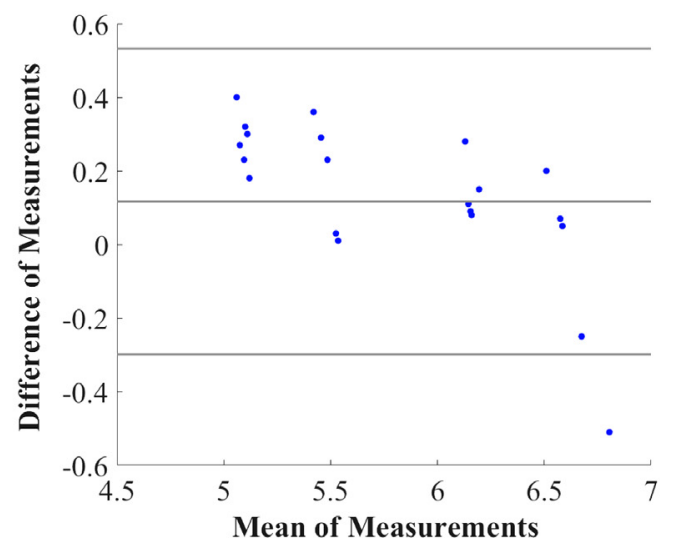

(b)

Fig. 6 (a) In vitro measurements: reference measurements using the conventional pH meter in green square, repeatable measurements using the FL system in blue circles, and error bars between average of the three repeated measurements and reference system in red. (b) Bland-Altman plot between reference $\mathrm{pH}$ meter and predicted $\mathrm{pH}$ values for in-vitro tooth samples (using $95 \%$ agreement between variables).

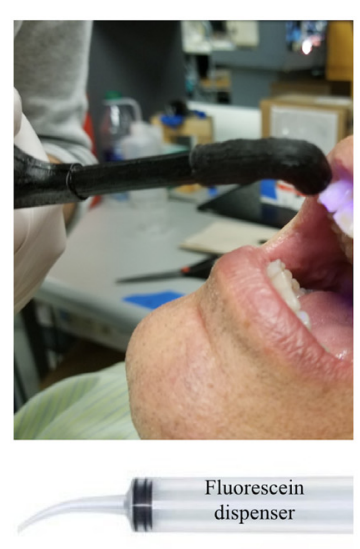

(a)

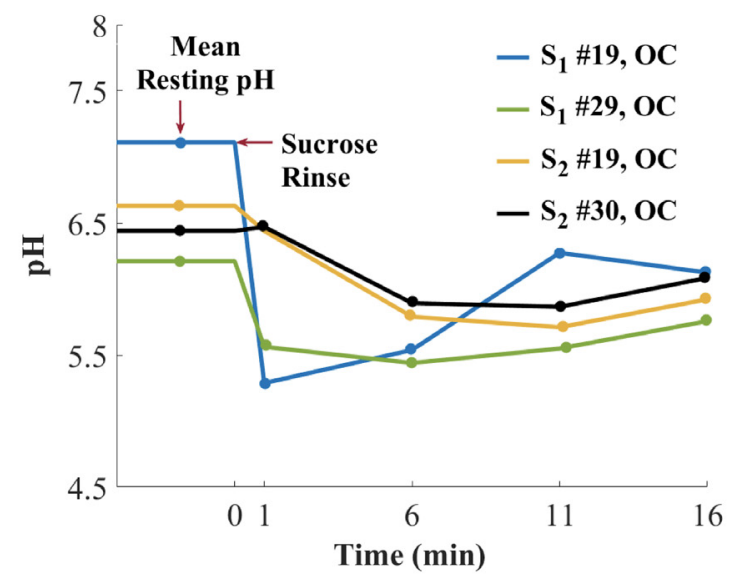

(b)

Fig. 7 (a) In vivo measurement and (b) mean resting $\mathrm{pH}$ and sucrose response of two subjects after $0.3-\mathrm{M}$ sucrose rinse. $\mathrm{pH}$ value before 0 min indicates mean resting $\mathrm{pH}$ of subject's tooth. Tooth number is listed in the legend along with the surface (OC: occlusal). The time from 1 to $16 \mathrm{~min}$ is the dynamic sucrose response obtained by taking measurements every $5 \mathrm{~min}$. 
A minute after the sucrose rinse, we measured the first $\mathrm{pH}$ value ( $1 \mathrm{~min})$ and then took three more measurements of selected tooth surface every $5 \mathrm{~min}$. As a control, buccal surface of tooth number 8 was selected with the assumption of low plaque load. Both resting and sucrose drop $\mathrm{pH}$ values were measured on the surface. After all the measurements, subjects' teeth were cleaned by a hygienist and evaluated by a dentist for low/ medium/high risk using CAMBRA (Caries Management by Risk Assessment). We had one high risk, one medium risk, and six low risk patients. All human subject involvement was approved under our University of Washington's IRB (IRB ID STUDY0004940).

Overall resting $\mathrm{pH}$ of 36 readings had one $\mathrm{SD}$ of $0.25 \mathrm{pH}$, four samples were removed due to absence of FL spectra reading. The average resting $\mathrm{pH}$ on the control tooth was 6.91 and average $\mathrm{pH}$ drop was of $0.4 \mathrm{pH}$ after the sucrose rinse. In six out of eight patients, we obtained $\mathrm{pH}$ drop of $0.4 \mathrm{pH}$ or more, within $5 \mathrm{~min}$ of the sucrose rinse, for at least one quadrant. Sucroseinduced $\mathrm{pH}$ response for the lower quadrants for the two subjects who skipped brushing are represented in Fig. 7. Occlusal (OC) surface was used for the results represented. $\mathrm{pH}$ region before $0 \mathrm{~min}$ indicates mean resting $\mathrm{pH}$ of the selected tooth. The time from 1 to $16 \mathrm{~min}$ is the dynamic sucrose response obtained by taking measurements every five minutes. The $\mathrm{pH}$ drop represented is more than $0.4 \mathrm{pH}$ for the four measurements depicted with a recovery trend toward the resting $\mathrm{pH}$. Lack of a larger, more significant number of patients prohibited a differentiation between low, medium, and high-risk patients on the basis of $\mathrm{pH}$ drop or resting $\mathrm{pH}$.

\section{Discussion}

The present device is able to track $\mathrm{pH}$ using only one fluorescent dye between 4.5 and 7 , ideal for measuring both resting and sucrose-induced plaque $\mathrm{pH}$ drop. The 420-nm LED was selected as the excitation source because its emission is absorbed by both the FL anion and dianion species. Furthermore, the two species exhibit distinctive spectral features that can be used for calibration and quantitative measurement of $\mathrm{pH}$. Verification results for the buffer solutions demonstrate that the system can measure $\mathrm{pH}$ values over a range of FL concentration. The unmixing algorithm uses normalized spectral data to derive ratiometric fractions of the anion and dianion species. This approach removes changes in the absolute amount of FL, which may be sensitive to photobleaching. Hence, the system is robust since the calibration only depends on the FL spectral band shapes.

Tests of buffered FL on extracted teeth demonstrate that the system can track the $\mathrm{pH}$ of FL on teeth with repeatable results, although the AF interference reduces the predicted accuracy in comparison to measurements taken in cuvette. Resting and sucrose rinse data in vivo indicate that the device has the capability to measure plaque $\mathrm{pH}$ in difficult to access regions such as occlusal pits and fissures. The optical probe design incorporates a replaceable hood that can either be disposed or sterilized between patient examinations. Replacing the 3-D-printed plastic with a smooth surfaced metal or injection molded plastic will further simplify cleaning of the device.

The pilot study highlighted further device improvements required for routine clinical use. The presence of ambient lighting and $\mathrm{AF}$ makes the $\mathrm{pH}$ measurement location dependent. The repeatability of locating the probe position between the AF, resting $\mathrm{pH}$, and sugar rinse measurement steps makes it difficult to mitigate the $\mathrm{AF}$ and ambient light noise. In an improved version of the device, a lock-in amplifier will be added to decrease interference of ambient light. Furthermore, a dye-in-polymer filter that selectively attenuates the ambient fluorescent light from 450 to $650 \mathrm{~nm}$ will be added to the probe. Though the AF interference was reduced by using higher FL concentration for in vivo testing, the spectral noise removal was susceptible to variation of illumination area within each measurement. This uncertainty can be alleviated by averaging multiple spots on a tooth surface or incorporating an imaging feature that will guide the probe. Other signal processing techniques such as machine learning can be added to improve accuracy and generate a heat map of plaque $\mathrm{pH}$.

The pilot study with low variability in measurement of resting $\mathrm{pH}$ shows potential of measuring in vivo $\mathrm{pH}$ values. The sucrose response shows that the device is capable of measuring in vivo the change of $\mathrm{pH}$ as a function of time. A longer-term clinical study is needed to determine whether a correlation can be established between susceptibility of caries and the time profile of the sucrose challenge. With only one high risk subject and absence of patients with active caries, it was difficult to draw any correlation between $\mathrm{pH}$ and caries detection. Also, most of the subjects had good dental hygiene, which lowered the accumulation of plaque, hindering the device functionality. The present study is also limited by the lack of a gold standard for measuring intraoral $\mathrm{pH}$. The $\mathrm{pH}$ microelectrode or even $\mathrm{pH}$ strips cannot be used on the irregular pits and fissures of occlusal surfaces. In future studies, we can measure the $\mathrm{pH}$ of scraped plaque of one location per patient using a conventional $\mathrm{pH}$ meter to compare with the optical method. However, the scraped plaque will be diluted, disturbed, and prone to sampling error-all of which could affect the $\mathrm{pH}$ measurement. In addition, in this study, the FL was not sufficiently viscous to stick to interproximal areas making it difficult to measure $\mathrm{pH}$ at those locations. Measurement of $\mathrm{pH}$ in the maxillary arch was facilitated by the use of a dental chair otherwise those locations would also suffer from lack of retention of FL. This retention of the FL solution can be improved by adding glycerol to the solution. In future studies, better control selection can be achieved by explicitly noting surfaces with lower plaque rather than using the same control for all the subjects.

A low-cost clinical device may require replacement of the spectrometer with multiple filtered photodiodes sensitive to selected FL emission wavelengths (e.g., 520 and $560 \mathrm{~nm}$ ). A similar FL intensity-based design was presented in a past work ${ }^{31}$ and can be modified to include only one LED excitation source and multiple filtered detectors or a detector array. With LED and photodiode components, the system could run on an embedded platform providing easier implementation of lock-in amplification and opportunity to develop a better user interface.

\section{Conclusion}

The noncontact, optical pH measurement system enables testing of plaque metabolism and is independent of specific species of dental biofilm. Furthermore, the device uses an FDA-approved FL dye that has been used by ophthalmologists at concentration levels over hundred times greater ${ }^{32}$ than our proposed method. The compact fiber optic-based device can be used to probe difficult to access occlusal and interproximal locations, paving the way for better understanding the correlation between plaque metabolic acidity and caries susceptibility. 


\section{Disclosures}

Authors MS, JYG, LYN, ZX, and EJS are coinventors of pending patent application that is owned by the University of Washington and is eligible to participate in a royalty sharing program when licensed.

\section{Acknowledgments}

This research was supported by the NSF PFI:BIC 1631146 Oral Health Monitor (PI: Seibel). We were also supported by the NSF REU and the Levinson Emerging Scholars Award. Clinical assistance was provided by Dr. Jacqueline Wong and Ms. Caroline Allen at the UW Dental School Clinic. We thank Dr. Alizera Sadr at the University of Washington for informative discussions on clinical translations of optical $\mathrm{pH}$ sensing in dentistry. We also thank Dr. Jeffrey McLean at the University of Washington and his lab for helpful discussions and creating human oral biofilms in vitro, which provided a foundation for this work.

\section{References}

1. P. D. Marsh, "Dental plaque as a biofilm and a microbial community: implications for health and disease," BMC Oral Health 6(Suppl. 1), S14 (2006).

2. N. Takahashi and B. Nyvad, "Caries ecology revisited: microbial dynamics and the caries process," Caries Res. 42(6), 409-418 (2008).

3. I. Kleinberg, "A mixed-bacteria ecological approach to understanding the role of the oral bacteria in dental caries causation: an alternative to Streptococcus mutans and the specific-plaque hypothesis," Crit. Rev. Oral Biol. Med. 13(2), 108-125 (2002).

4. J. D. B. Featherstone and B. E. Rodgers, "Effect of acetic, lactic and other organic acids on the formation of artificial carious lesions," Caries Res. 15(5), 377-385 (1981).

5. W. Krzyściak et al., "The virulence of Streptococcus mutans and the ability to form biofilms," Eur. J. Clin. Microbiol. Infect. Dis. 33(4), 499-515 (2014)

6. C. Dawes, "What is the critical $\mathrm{pH}$ and why does a tooth dissolve in acid?" J. Can. Dent. Assoc. 69(11), 722-725 (2003).

7. R. M. Stephan, "Intra-oral hydrogen-ion concentrations associated with dental caries activity," J. Dent. Res. 23(4), 257-266 (1944).

8. R. H. Selwitz, A. I. Ismail, and N. B. Pitts, "Dental caries," Lancet 369(9555), 51-59 (2007).

9. P. D. Marsh, "Are dental diseases examples of ecological catastrophes?" Microbiology 149(2), 279-294 (2003).

10. J. Xiao et al., "Biofilm three-dimensional architecture influences in situ $\mathrm{pH}$ distribution pattern on the human enamel surface," Int. J. Oral Sci. 9(2), 74-79 (2017)

11. S. Hata and H. Mayanagi, "Acid diffusion through extracellular polysaccharides produced by various mutants of Streptococcus mutans," Arch. Oral Biol. 48(6), 431-438 (2003).

12. J. Hicks, F. Garcia-Godoy, and C. Flaitz, "Biological factors in dental caries: role of saliva and dental plaque in the dynamic process of demineralization and remineralization (part 1)," J. Clin. Pediatr. Dent. 28(1), 47-52 (2004)

13. J. Hicks, F. Garcia-Godoy, and C. Flaitz, "Biological factors in dental caries enamel structure and the caries process in the dynamic process of demineralization and remineralization (part 2)," J. Clin. Pediatr. Dent. 28(2), 119-124 (2005).

14. G. Sharma, M. P. Puranik, and K. R. Sowmya, "Approaches to arresting dental caries: an update," J. Clin. Diagn. Res. 9(5), ZE08-ZE11 (2015).

15. E. M. Aranibar Quiroz et al., "Relationship between plaque $\mathrm{pH}$ and different caries-associated variables in a group of adolescents with varying caries prevalence," Caries Res. 48(2), 147-153 (2014).

16. Y. M. Dong et al., "Plaque $\mathrm{pH}$ and associated parameters in relation to caries," Caries Res. 33(6), 428-436 (1999).

17. O. Fejerskov, A. A. A. Scheie, and F. Manji, "The effect of sucrose on plaque $\mathrm{pH}$ in the primary and permanent dentition of caries-inactive and -active Kenyan Children," J. Dent. Res. 71(1), 25-31 (1992).
18. P. Lingström et al., " $\mathrm{pH}$ measurements of human dental plaque after consumption of starchy foods using the microtouch and the sampling method," Caries Res. 27(5), 394-401 (1993).

19. S. R. Wood et al., "Architecture of intact natural human plaque biofilms studied by confocal laser scanning microscopy," J. Dent. Res. 79(1), 21-27 (2000).

20. C. M. C. Volgenant et al., "Red fluorescent biofilm: the thick, the old, and the cariogenic," J. Oral Microbiol. 8(1), 30346 (2016).

21. A. Carlén, H. Hassan, and P. Lingström, "The 'strip method': a simple method for plaque $\mathrm{pH}$ assessment," Caries Res. 44(4), 341-344 (2010).

22. J. Guo et al., "Plastic reusable $\mathrm{pH}$ indicator strips: preparation via anionexchange of poly(ionic liquids) with anionic dyes," Polym. Chem. 4(5), 1309-1312 (2013).

23. S. Schlafer and R. L. Meyer, "Confocal microscopy imaging of the biofilm matrix," J. Microbiol. Methods 138, 50-59 (2017).

24. D. E. Caldwell, D. R. Korber, and J. R. Lawrence, "Imaging of bacterial cells by fluorescence exclusion using scanning confocal laser microscopy," J. Microbiol. Methods 15(4), 249-261 (1992).

25. R. Sjöback, J. Nygren, and M. Kubista, "Absorption and fluorescence properties of fluorescein," Spectrochim. Acta Part A Mol. Biomol. Spectrosc. 51(6), L7-L21 (1995).

26. N. Klonis and W. H. Sawyer, "Spectral properties of the prototropic forms of fluorescein in aqueous solution," J. Fluoresc. 6(3), 147157 (1996).

27. M. Kubista, R. Sjoback, and B. Albinsson, "Determination of equilibrium constants by chemometric analysis of spectroscopic data," Anal. Chem. 65(8), 994-998 (1993).

28. T. C. O'Haver, "Teaching and learning chemometrics with MatLab," Chemom. Intell. Lab. Syst. 6(2), 95-103 (1989).

29. J. S. McLean et al., "Identifying low $\mathrm{pH}$ active and lactate-utilizing taxa within oral microbiome communities from healthy children using stable isotope probing techniques," PLoS One 7(3), e32219 (2012).

30. H. Jeong et al., "Indium gallium nitride-based ultraviolet, blue, and green light-emitting diodes functionalized with shallow periodic hole patterns," Sci. Rep. 7(March), 1-9 (2017).

31. M. Sharma et al., "Dental pH Opti-Wand (DpOW): measuring oral acidity to guide enamel preservation," Conf. Proc. IEEE Eng. Med. Biol. Soc. 2018, 3738-3741 (2018)

32. Alcon, "Ophthalmic disclosing agent fluorescein sod," Mckesson Medical-Surgical Inc, 2018, https://mms.mckesson.com/product/ 897229/Alcon-00065009265.

Manuja Sharma is a second year PhD student in electrical and computer engineering at the University of Washington, Seattle. She received her bachelor's degree in electrical engineering from the University of Washington in 2017. Her research interests include developing ubiquitous health sensing applications.

Jasmine Y. Graham is a PhD student in the University of California, Berkeley-University of California, San Francisco Graduate Program in Bioengineering. She received her BS degree in bioengineering from the University of Washington and developed an optical measurement of acidification in vitro to predict dental caries in the University of Washington Human Photonics Laboratory. Her research interests include developing and translating diagnostic technology to clinical practice.

Philip A. Walczak is a second year dental student in the DDS/PhD program at the University of Washington School of Dentistry. He has four years of experience in dental research, with previous work on dental material properties, implant stability testing device development, and clinical testing. His interests mainly focus around prevention and utilizing biological responses to prevent disease development, in addition to increasing access to care.

Ryan Nguyen is a senior undergraduate student in microbiology at the University of Washington. His research interests include biofilm analysis and development of dental tools for better diagnostics.

Lauren K. Lee is currently a predental student in her fourth undergraduate year at the University of Washington. Her main areas of research interest include the detection and prevention of dental caries, and relevant public education. 
Matthew D. Carson is a staff engineer with HPL. He earned his BS degree in physics from the UW. He brings to HPL the experience of working in a broad variety of commercial startups pursuing innovative technologies.

Leonard Y. Nelson received his undergraduate and $\mathrm{PhD}$ degrees in chemistry from Rensselaer Polytechnic Institute and the University of California, Berkeley, respectively. His graduate research dealt with infrared spectroscopic studies of rare gas halogen molecules condensed at cryogenic temperatures. Following completion of his graduate research, he worked in advanced laser technology (10 years) and cockpit display systems (25 years). After retirement, he joined the HPL group to mentor and collaborate with students in projects related to chemistry and spectroscopy.

Shwetak N. Patel is the Washington Research Foundation Entrepreneurship Endowed Professor in computer science and engineering and electrical engineering at University of Washington, Seattle. He received his $\mathrm{PhD}$ in computer science from Georgia Institute of Technology in 2008 and his BS in computer science in 2003. His research interests are in the areas of human-computer interaction, ubiquitous computing, sensor-enabled embedded systems, and user interface software and technology.

Zheng $\mathbf{X u}$ is a clinical associate professor in pediatric dentistry at the University of Washington. She is the program director for the advanced education program in pediatric dentistry. She is a consultant for the American Board of Pediatric Dentistry and has served on the oral clinical examination subcommittee since 2014. She also is a member of the editorial board of pediatric dentistry, the official journal of the American Academy of Pediatric Dentistry.

Eric $\mathbf{J}$. Seibel is a research professor of mechanical engineering, adjunct in bioengineering, electrical engineering, oral health sciences, and director of the Human Photonics Laboratory at the University of Washington. He received his $\mathrm{BS}$ and MS degrees in mechanical engineering from Cornell University and University of California, Berkeley, respectively, and his $\mathrm{PhD}$ in bioengineering from the University of Washington, Seattle. His expertise is bringing new optomechanical biomedical devices from bench to bedside, such as the scanning fiber endoscope. He is a senior member of SPIE. 